\title{
Geographical proximity of takeaway food outlets to schools, colleges and universities in a low-socioeconomic ward in Manchester
}

\author{
J. Blow, S. Patel and R. Gregg \\ Department of Health Professions, Manchester Metropolitan University, Manchester, M15 6BG
}

The socioeconomic disparity in the prevalence of childhood and early adult obesity has been well characterised ${ }^{(1)}$. Currently, takeaway and fast foods make up a considerable proportion of the UK diet. However, not only do these foods have a poor nutritional profile ${ }^{(2)}$, the food outlets, from which they are purchased are thought to cluster in lower socioeconomic areas ${ }^{(3)}$. In addition there is greater concern of their proximity to schools ${ }^{(4)}$ and thus the physical food environment that students are exposed to. This study aimed to map takeaway food outlets and their proximity to schools, colleges and universities in order to characterise the physical takeaway food environment within a low-socioeconomic ward in Manchester.

The defined ward, along with a $2 \mathrm{~km}$ Euclidean buffer radius, was included as this was considered within delivery distance. Takeaway food was defined as convenience and fast food meals purchased from small, independent outlets, that are commercially pre-prepared and ready for immediate consumption, either eaten in-store or elsewhere, or ordered for delivery ${ }^{(5)}$. Data from the local authority on food businesses classed as hot food takeaways, and Environmental Health register on food hygiene ratings were used to map the physical environment of takeaway outlets, using QGIS version 2.18.0 (OPENGIS.ch LLC, Einsiedeln, Switzerland). Education establishments (excluding adult learning facilities) were located within the study area, using Ordinance Survey Points of Interest, and number of outlets within a $400 \mathrm{~m}$ Euclidean walking buffer were mapped.

Within the study area, 202 takeaway food outlets were identified and mapped as cluster points (Fig 1a). Of these $62 \cdot 3 \%$ are located on $\mathrm{A}$ and $\mathrm{B}$ roads, whilst the remaining outlets were located on minor roads.

Of the 53 schools, colleges and universities within the study area, $12(22.6 \%)$ had 0 takeaway outlets within $400 \mathrm{~m}, 37(69.8 \%)$ had 1-10 takeaway outlets within $400 \mathrm{~m}$, and 1 (1.9\%) had 21-30 outlets within $400 \mathrm{~m}$ (Fig 2b).
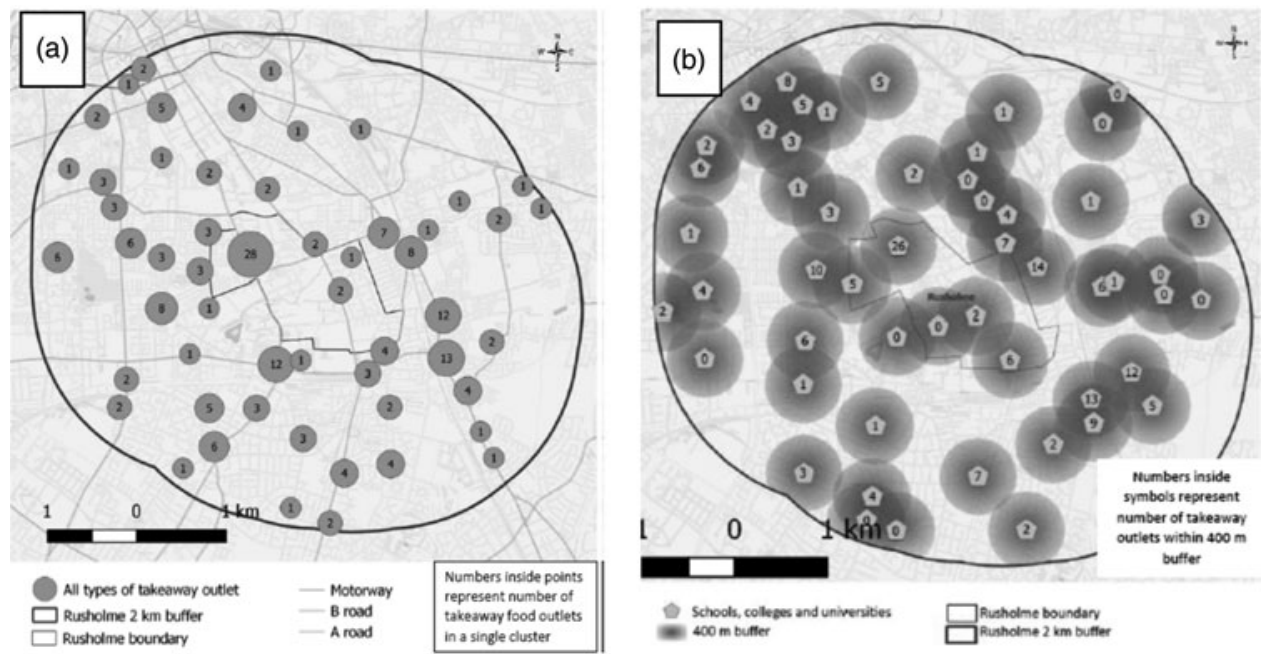

Fig. 1. Cluster map of takeaway food outlets (a) and the number of takeaways outlets all schools, colleges and universities with $400 \mathrm{~m}$ buffers (b) within the Rusholme $+2 \mathrm{~km}$ buffer area

Within this low-socioeconomic area, there was a high concentration of takeaway food outlets, predominantly along major roads. In addition, the majority of these outlets were in easy walking distance of educational establishments. This work informs the need for stricter planning laws to restrict the proliferation of takeaway outlets. This could help to improve the physical food environment and thus reduce the socioeconomic disparity in obesity.

1. Stamatakis E, Wardle J, Cole TJ (2010) Int J Obs 34, 41-47.

2. Smith KJ, McNaughton SA, Gall SL et al. (2009) Int J Behav Nutr Phys Act 6, 29.

3. Fraser LK, Edwards KL, Cade J, C et al. (2010) Int J Environ Res Public Health 7, 2290-2308.

4. Day PL, Pearce J (2011) Am J Prev Med 40, 113-121.

5. Jaworowska A, Blackham T, Stevenson L et al. Appetite 59, 517-522. 